\title{
Influence of structural parameters on pitching stability of a vertical shiplift
}

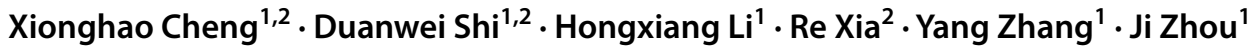

Received: 19 November 2019 / Accepted: 8 August 2020 / Published online: 18 August 2020

(c) Springer Nature Switzerland AG 2020

\begin{abstract}
To analyze the pitching stability of the fully balanced hoist vertical shiplift, this paper develops a fluid velocity potential and dynamical model of the vertical shiplift based on an improved energetic exponent method, to study the influence of the primary structural parameters on the pitching stability of an actual shiplift installed in GuoPitan shiplift (GuiZhou province, China). With the linearized potential theory and the undetermined coefficient method, the numerical analytical expression of the velocity potential is obtained by discussing the pitching motion of ship chamber. Applying the Lagrange equation of the second kind to establish a dynamical model of the shiplift having multiple degrees of freedom, the energetic calculated model for ship chamber in respect to the pitching motion is proposed. To accurately analyze the variation of the pitching stability, this paper develops the slope of energetic varied curve, based on the orthogonal polynomial approximation and the subsection averaging method. Also, the influence of the parameters is studied, including the distance of lifting point, the synchronous synchronous shaft stiffness, and the hoist radius, with the optimized design of these three structural parameters: $a=40.0 \mathrm{~m}, C=2.0 \times 10^{9} \mathrm{~N} / \mathrm{m}$, and $R=1.8 \mathrm{~m}$. The changing range of the absolute value of energetic exponent for the distance of lifting points is the largest of the three structural parameters (5.00-12.80), indicating that the distance of lifting points has the most remarkable influence on the pitching stability. Moreover, by the Runge-Kutta algorithm, the response of the angle displacement is analyzed before and after optimization, and then the reliability of results is demonstrated. The four angle displacements at the peak values close to $300 \mathrm{~s}$ decrease by $11.11 \%, 13.33 \%, 15.91 \%$, and $10.86 \%$ respectively after optimization. It reveals that the pitching stability of shiplift is enhanced evidently after optimization.
\end{abstract}

Keywords Energetic exponent method - Fully balanced hoist vertical shiplift - Optimization · Pitching stability . Structural parameter

\section{Introduction}

Lately in China, there has been a huge demand for water conservancy projects and their related innovations. Under these social circumstances, the paper aims to provide some technical advises for the second step of GuoPitan Shiplift, mainly focusing on the pitching stability. Full balanced hoist vertical shiplift, a kind of navigation structure, is used for ship to overcome the difference of water level between the upstream and downstream of water conservancy project. As is shown in Fig. 1, the primary structure of full balanced hoist vertical shiplift is composed of ship chamber, hoisting system and tower column. More specifically, the part A in Fig. 1 shows that the hoisting system consists of winding drums lifting ship chamber,and synchronous shaft making the lifting of four winding drums

Duanwei Shi, dwshi@whu.edu.cn | ${ }^{1}$ Hubei Key Laboratory of Accoutrement Technique in Fluid Machinery and Power Engineering, Wuhan, People's Republic of China. ${ }^{2}$ School of Power and Mechanical Engineering, Wuhan University, Wuhan 430072 , People's Republic of China. 


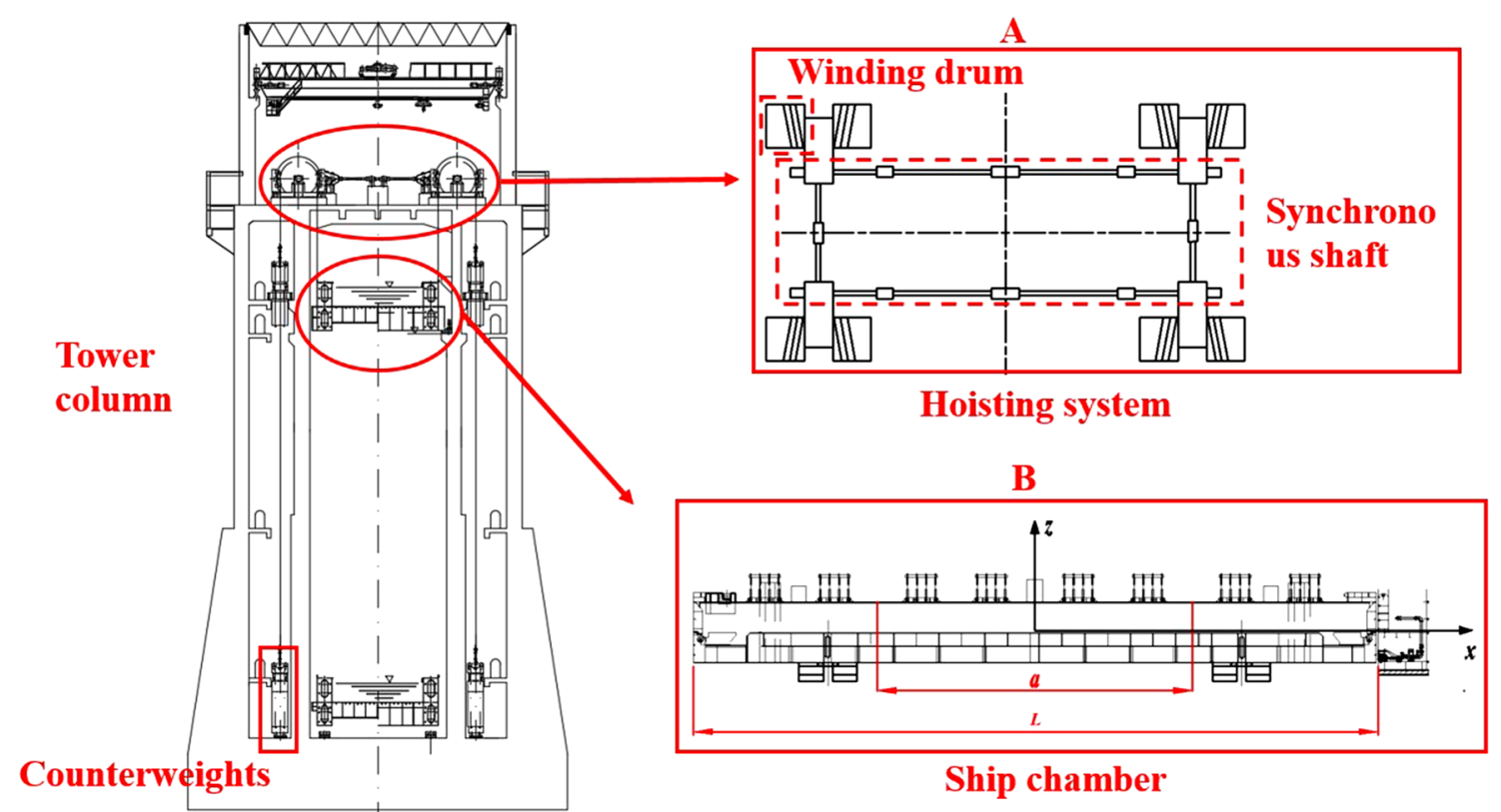

Fig. 1 Primary structure of full balanced hoist vertical shiplift

as synchronous as possible. The part B in Fig. 1 shows that the cross-sectional view of ship chamber, $a$ is the distance of two lifting points and one lifting point is formed by each two winding drums in one side of ship chamber. Counterweights include gravity counterweights connected to ship chamber with wire ropes, and torque counterweights connected to winding drums with wire ropes. In this paper, the primary three parameters, including distance of two lifting points $a$, stiffness of synchronous shaft $C$, and radius of winding drum $R$, are the keys of discussion.

In a full balanced hoist vertical shiplift, with the closed rectangular synchronous shafts, long and narrow ship chamber, and the larger elasticity of wire ropes, it is difficult to accurately quantify the various influential factors for pitching stability and their influential degree [1-3]. If the ship chamber capsizes due to instability, it will cause huge economic loss and intolerable social impact. Therefore, with the aim of distinguishing the parameters with great influence on pitching stability, it is very significant to study the accurate quantitative characterization for the influence of structural parameters on pitching stability.

Regarding the pitching stability of ship chamber, a discriminant of instability condition for ship chamber was derived based on the static equilibrium, with respect to water leakage condition [4]. The natural vibration characteristics and stability of full balanced hoist vertical shiplift, containing ship chamber, wire ropes, gravity counterweights, water and ship in ship chamber, was studied to develop the critical influential factors for natural vibration characteristics as well as the critical distance of lifting points maintaining stability $[5,6]$. The influence of synchronous shaft in the main lifting system was neglected in all the above studies. By establishing a series of the multivariable nonlinear differential equations, and using numerical calculation based on the Runge-Kutta method, the stability of ship chamber subjected to the rigid vertical vibration was addressed $[7,8]$. A theoretical formulation was developed to analyze the critical distance of lifting points, by establishing a dynamical model without gravity counterweights [9]. Assuming a time-harmonic pitching excitation, a fluid dynamic model is presented to predict the capsizing moments based on the Housner theory [10]. A semi-analytical scheme is proposed to analyze the pitching stability of the high-lift wire ropes vertical shiplift [11]. In this study, a new 9-DOF coupled dynamics model of the complete main hoist system, shiplift chamber motion and shallow water sloshing was established. Combining the Lyapunov motion stability theory, the critical distance of suspension points (the important indicator of pitch stability) would be numerically calculated. When it comes to the theoretical studies about stability, there are many mathematical analytical methods including the Routh-Hurwitz theory, Lyapunov stability theory, Floquet theory, and Mathieu equation etc. More specifically, Lyapunov stability theory is suitable only for linear and nonlinear systems, rather than multi-free linear time-invariant systems, timevarying linear systems and nonlinear systems [12]. The Floquet theory and Mathieu equation are usually applied to study the stability of solutions for linear homogeneous periodic coefficient differential equations [13-15]. As for 
constant coefficient linear system, a characteristic equation can be obtained by transforming coefficient matrix of differential equations. Under the Routh-Hurwitz theory, stability condition can be obtained in accordance with the root of the characteristic equation $[16,17]$. However, without intuitive characterization of the changing law of stability, the stability criterion derived from the above theoretical analysis is considerably complicated. In terms of the stability of parametrically excited vibration systems, there are several primary research methods including Bolotin method, perturbation method, multiscale method, and numerical continuation method, etc. [18-20]. With drawing method, these works have been devoted to identify the unstable regions and to predict the possibility of instability. Nevertheless, it is exceedingly difficult to identify the unstable region through this method, because the instability under different incentives is highly different for a great number of nonlinear systems. Therefore, an increasing number of researchers have conducted numerous works about innovative methods of evaluating instability. The growth rate of instability characteristic is evaluated by the exponent in Floquet theory [21, 22]. Subsequently, an energetic exponent method was firstly proposed to analyze the stability of two-dimensional fluid sloshing in the container of various shape, and the mathematical model of energetic exponent is derived based on Mathieu equation $[23,24]$. These two papers indicate that the energetic exponent method is very convenient and efficient to accurately quantify the stability of two dimensional fluid-solid coupling system by analyzing the mechanical energy of system. However, the traditional energetic exponent method cannot present the change of the pitching stability directly because of its nonlinear characteristic. In order to compensate for this weakness, an improved energetic exponent method is proposed to perform an approximation solution of linear polynomial for each part of the slope of energetic exponent curve, by using the Best square approximation method for Legendre orthogonal polynomials as well as the subsection averaging method.

Section 3 of this paper derives a mathematical model for velocity potential of fluid subjected to pitching excitation. Section 4 proposes an improved energetic exponent method based on the dynamical model of shiplift. In Sect. 5, it investigates the influence of the three structural parameters with the improved energetic exponent method. As for Sect. 6, the three structural parameters of the second step of the shiplift of GouPitan water conservancy is optimized to increase the stability, and a formulation is proposed to analyze the change of the stability. Finally, a set of numerical results is obtained by using the Runge-Kutta algorithm, and the comparison of the numerical and the theoretical results proves the validity of the theoretical analysis.

\section{Velocity potential of water in ship chamber}

Figure 2 illustrates that the ship chamber of the full balanced hoist vertical shiplift is a two dimensional rectangular container filled with fluid, $h$ means the depth of fluid and $L$ means the length of ship chamber. It is assumed that the fluid is incompressible and inviscid, and the coordinate system origin $O$ is located at the height of $h / 2$, with $\phi$ being the fluid velocity potential. Thus, the fluid continuity equation is as follows:

$\frac{\partial^{2} \phi}{\partial x^{2}}+\frac{\partial^{2} \phi}{\partial z^{2}}=0$

The boundary conditions of the free surface are written as:

$\left.\left(\frac{\partial^{2} \phi}{\partial t^{2}}+g \frac{\partial \phi}{\partial z}\right)\right|_{z=\frac{h}{2}}=0$

where $t$ means time. Assuming $a(t)$ is the pitching motion of the ship chamber boundaries, it can be given by:

$\left.\frac{\partial \phi}{\partial z}\right|_{z=-\frac{h}{2}}=-x \dot{\alpha}(t)$

$\left.\frac{\partial \phi}{\partial x}\right|_{x= \pm \frac{L}{2}}=z \dot{\alpha}(t)$

The assumption of velocity potential [18] is written as:

$\phi=\sum_{n=1}^{\infty} A_{n} \sinh \left[\frac{(2 n-1) \pi z}{h}\right] \sin \left[\frac{(2 n-1) \pi x}{h}\right]$

where $A_{n}$ is the amplitude of velocity potential. Next, the Fourier series expansion is carried out for $x$ in Eq. (3), and set $\lambda_{n}=(2 n-1) \pi$, the equation can be written as:

$x=\sum_{n=1}^{\infty}\left(\frac{2 h^{2}}{\lambda_{n}^{2}}\right)(-1)^{n-1} \sin \left(\frac{\lambda_{n} x}{h}\right)$.

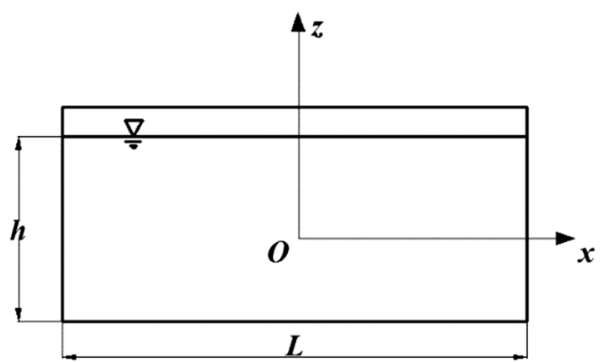

Fig. 2 2-D water area for ship chamber 
Based on Eqs. (3), (5) and (6), the equation is as follows as:

$\sum_{n=1}^{\infty} A_{n} \frac{\lambda_{n}}{h} \cosh \left(\frac{\lambda_{n}}{2}\right) \sin \left(\frac{\lambda_{n} x}{h}\right)=-\sum_{n=1}^{\infty}\left(\frac{2 h^{2}}{\lambda_{n}^{2}}\right)(-1)^{n-1} \sin \left(\frac{\lambda_{n} x}{h}\right) \dot{\alpha}(t)$.

Thus, time function $A_{n}$ is written as:

$A_{n}=2(-1)^{n}\left(\frac{h}{\lambda_{n}}\right)^{3} \frac{\dot{\alpha}(t)}{\cosh \left(\frac{\lambda_{n}}{2}\right)}$.

The $A_{n}$ be obtained by substituting Eq. (8) into Eq. (5), in the form:

$\phi=\sum_{n=1}^{\infty} \frac{\dot{\alpha}(t)\left(2 h^{3}\right)(-1)^{n}}{\cosh \left(\frac{\lambda_{n}}{2}\right) \lambda_{n}^{3}} \sinh \left(\frac{\lambda_{n} z}{h}\right) \sin \left(\frac{\lambda_{n} x}{h}\right)$.

\section{Improved energetic exponent method}

\subsection{Formulation of energetic exponent}

The energetic expression for pitching motion of ship chamber is written as [24]:

$T(t)=\iiint \frac{\rho}{2}\left\{\left[\frac{\partial \phi(x, z)}{\partial x}\right]^{2}+\left[\frac{\partial \phi(x, z)}{\partial z}\right]^{2}\right\} d x d y d z$

$V(t)=B \int_{\partial S_{f}} \frac{\rho g}{2} z^{2}(x, t) d s$

where $T(t)$ and $V(t)$ are the kinetic energetic and potential energy of the fluid system, respectively. $B$ is the width of ship chamber, $\rho$ is the density of liquid, $g$ is acceleration of gravity.

Based on Eqs. (9), (10) and (11), the function of energetic varies with time $E(t)$ can be obtained, in the form:

$E(t)=T(t)+V(t)=I_{1}[\dot{q}(t)]^{2}+I_{2}[q(t)]^{2}$

where $I_{1}(i=1,2), q(t)$ and $q(t)$ can be calculated as follows:

$I_{1}=\frac{B \rho \pi(-1+2 n)\left(h \sin \left[\frac{L(1-2 n) \pi}{h}\right]-L \sinh [(1-2 n) \pi]\right)}{4 h}$

$I_{2}=-\frac{(-1+2 n) \pi \cosh \left[\frac{\pi-2 n \pi}{h}\right]^{2}\left(L(-1+2 n) \pi+h \sinh \left[\frac{L(1-2 n) \pi}{h}\right]\right) \rho g B}{4 h^{2}}$
$\dot{q}(t)=A_{n}$

$q(t)=\frac{\alpha(t)\left(2 h^{3}\right)(-1)^{n}}{\cosh \left(\frac{\lambda_{n}}{2}\right) \lambda_{n}^{3}}$.

The formulation of energetic exponent $F(t)$ is arranged in the form:

$F(t)=\ln \left[\frac{E\left(t, \xi_{1}\right)}{E\left(0, \xi_{1}\right)}\right]$.

\subsection{Dynamic model of vertical shiplift}

Vertical shiplift, most commonly used form of shiplift, has been widely used in China where seven vertical shiplifts are functioning for primary water conservancy projects. GouPitan shiplift, discussed in this paper, is most representative vertical shiplift. Figure 3 illustrates the dynamic model of fully balanced hoist vertical shiplift, with nine degrees of freedom together: $a(t)$ being pitching angle displacement of ship chamber, $\theta(t)$ being pitching angle displacement of free surface, $\varphi_{1}(t)$ and $\varphi_{2}(t)$ being rotating angle displacement of hoist, $z_{1}(t)$ and $z_{4}(t)$ being vertical displacement of the torque counterweights, $z_{2}(t)$ and $z_{3}(t)$ being vertical displacement of the gravity counterweights, $z_{5}(t)$ being vertical displacement of the ship chamber. $J_{1}$ denotes the equivalent moment of inertia for the hoist and torque counterweights, with $J_{2}$ being the equivalent moment of inertia for the pulleys, $J_{3}$ being the equivalent moment of inertia for the pitching motion of the ship chamber and liquid, $\gamma_{i}(i=1,2,3)$ respectively are the damping coefficient of the torsional vibration of the winding

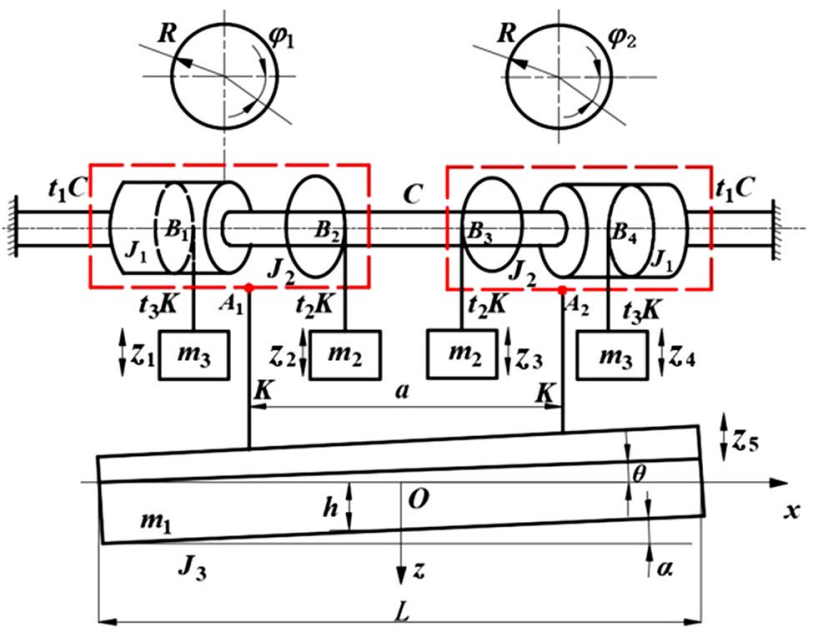

Fig. 3 Model diagram of the full balanced hoist vertical shiplift 
drums, the damping coefficient of the pitching motion for ship chamber and the damping coefficient of the vertical motion for ship chamber. $K$ being the stiffness of the wire ropes, $C$ being the stiffness of the synchronous shaft, $R$ being the radius of the winding drums, $t_{i}(i=1,2,3)$ being the proportional coefficient of wire ropes and synchronous shaft, $m_{1}$ being the mass of liquid and ship chamber, $m_{2}$ being the mass of gravity counterweights, $m_{3}$ being the mass of gravity counterweights, $F$ being the loading of ship chamber in the vertical direction.

For the convenience of solution, each free degree can be written as:

$$
\begin{aligned}
& m_{2} \ddot{z}_{3}+\gamma_{3} \dot{z}_{3}+t_{2} K\left(R \varphi_{2}+z_{3}\right)=0 \\
& m_{3} \ddot{z}_{4}+\gamma_{3} \dot{z}_{4}+t_{3} K\left(R \varphi_{2}+z_{4}\right)=0 \\
& m_{1} \ddot{z}_{5}+\gamma_{3} \dot{z}_{5}-K\left(R \varphi_{1}-z_{5}-\frac{1}{2} a \alpha\right)-K\left(R \varphi_{2}-z_{5}+\frac{1}{2} a \alpha\right)=F
\end{aligned}
$$

where $J_{s}=(1 / 24) \rho B h L^{3}, C_{s}=(1 / 12) \rho B h L^{3}$.

$\left[\alpha(t), \theta(t), \varphi_{1}(t), \varphi_{2}(t), z_{1}(t), z_{2}(t), z_{3}(t), z_{4}(t), z_{5}(t)\right]=e^{\xi_{i} t} \sin t,(i=1,2, \ldots, 9)$.

The kinetic energy and potential energy of shiplift is

defined as:

$$
\begin{aligned}
T= & \frac{1}{2} J_{1}\left(\dot{\varphi}_{1}^{2}+\dot{\varphi}_{2}^{2}\right)+\frac{1}{2} J_{2}\left(\dot{\varphi}_{1}^{2}+\dot{\varphi}_{2}^{2}\right)+\frac{1}{2} J_{3} \dot{\alpha}^{2}+\frac{1}{2} m_{1} \dot{z}_{5}^{2}+\frac{1}{2} m_{2} \dot{z}_{2}^{2}+\frac{1}{2} m_{2} \dot{z}_{3}^{2}+\frac{1}{2} m_{3} \dot{z}_{1}^{2}+\frac{1}{2} m_{3} \dot{z}_{4}^{2} \\
V= & \frac{1}{2} K\left(R \varphi_{1}-z_{5}-\frac{1}{2} a \alpha\right)^{2}+\frac{1}{2} K\left(R \varphi_{2}-z_{5}+\frac{1}{2} a \alpha\right)^{2}+\frac{1}{2} t_{3} K\left(R \varphi_{1}+z_{1}\right)^{2}+\frac{1}{2} t_{2} K\left(R \varphi_{1}+z_{2}\right)^{2} \\
& +\frac{1}{2} t_{2} K\left(R \varphi_{2}+z_{3}\right)^{2}+\frac{1}{2} t_{3} K\left(R \varphi_{2}+z_{4}\right)^{2}+\frac{1}{2} C\left(\varphi_{1}-\varphi_{2}\right)^{2}+\frac{1}{2} t_{1} C\left(\varphi_{1}^{2}+\varphi_{2}^{2}\right) .
\end{aligned}
$$

Using the Lagrange equation of the second kind, the dynamical equations are as follows:

$$
\begin{aligned}
& \left(J_{3}+2 J_{s}\right) \ddot{\alpha}+\gamma_{2} \dot{\alpha}-\frac{1}{2} a K\left(R \varphi_{1}-z_{5}-\frac{1}{2} a \alpha\right)+\frac{1}{2} a K\left(R \varphi_{2}-z_{5}+\frac{1}{2} a \alpha\right)-C_{s}(\alpha-\theta)-\frac{10 g h}{L^{2}} J_{s} \theta=0 \\
& \left(J_{3}+2 J_{s}\right) \ddot{\theta}+\gamma_{2} \dot{\alpha}-\frac{1}{2} a K\left(R \varphi_{1}-z_{5}-\frac{1}{2} a \alpha\right)+\frac{1}{2} a K\left(R \varphi_{2}-z_{5}+\frac{1}{2} a \alpha\right) \\
& -C_{s}(\alpha-\theta)+\frac{10 g h}{L^{2}}\left(J_{s}+J_{3}\right) \theta=0
\end{aligned}
$$

$$
\begin{aligned}
& \left(J_{1}+J_{2}\right) \ddot{\varphi}_{1}+\gamma_{1} \dot{\varphi}_{1}+K R\left(R \varphi_{1}-z_{5}-\frac{1}{2} a \alpha\right)+t_{3} K R\left(R \varphi_{1}+z_{1}\right) \\
& +t_{2} K R\left(R \varphi_{1}+z_{2}\right)+C\left(\varphi_{1}-\varphi_{2}\right)+t_{1} C \varphi_{1}=0
\end{aligned}
$$$$
\left(J_{1}+J_{2}\right) \ddot{\varphi}_{2}+\gamma_{1} \dot{\varphi}_{2}+K R\left(R \varphi_{2}-z_{1}+\frac{1}{2} a \alpha\right)+t_{2} K R\left(R \varphi_{2}+z_{3}\right)
$$$$
+t_{3} K R\left(R \varphi_{2}+z_{4}\right)-C\left(\varphi_{1}-\varphi_{2}\right)+t_{1} C \varphi_{2}=0
$$$$
m_{3} \ddot{z}_{1}+\gamma_{3} \dot{z}_{1}+t_{3} K\left(R \varphi_{1}+z_{1}\right)=0
$$$$
m_{2} \ddot{z}_{2}+\gamma_{3} \dot{z}_{2}+t_{2} K\left(R \varphi_{1}+z_{2}\right)=0
$$

\subsection{Slope of energetic exponent curve exponent}

A series of nonlinear equations with respect to $\xi_{i}(i=1$, $2, \ldots, 9)$ can be elicited by combining Eqs. (14) and (16). Subsequently, with the application of Broyden quasi-Newton method [8], $\xi_{1}$ and $a(t)$ are obtained. Finally, $F\left(t, \xi_{1}\right)$ is spelled out based on the Eqs. (12d) and (13).

The slope of energetic exponent curve cannot present the change of the pitching stability directly because of its nonlinear characteristic. In order to compensate for this weakness, an improved energetic exponent method is proposed to perform an approximation solution of linear polynomial for the slope of energetic exponent curve, by 
using the Best square approximation method for Legendre orthogonal polynomials as well as the subsection averaging method. As is shown in Fig. 4, beginning from [0, 1], the interval is narrowed continuously by changing its right endpoint until the approximation error of linear polynomial is lower than the controlling range, and the slope of this linear polynomial at this time is named as $K_{1}$. Next, it is essential that the right endpoint is transformed into the left endpoint of the next interval, and a new interval is obtained by repeating the previous steps until the left endpoint of the $\mathrm{N}$ interval becomes 100, with attaining a series of the slopes $K_{2}, K_{3}, \ldots, K_{n}$.

Subsequently, the slope of energetic exponent for vertical shiplift $K$ is written as:

$K=\frac{1}{n} \sum_{i=1}^{n} K_{i}$

Finally, the criteria for stability is arranged in the form:

$K=\left\{\begin{array}{l}>0 \text { stability } \\ =0 \text { stability bondary } . \\ <0 \text { instability }\end{array}\right.$

\section{Influence of structural parameters}

To analyze the influence of the primary structural parameters including the distance of critical lifting point $a$, the synchronous shaft stiffness $C$, as well as the hoist radius $R$, taking the second step of shiplift of GouPitan water conservancy, with the greatest lifting height and lifting mass $\left(H=113.0 \mathrm{~m}, m_{1}=m_{2}=1075 t\right)$ throughout China, as a sample calculation. Founded on the design experience

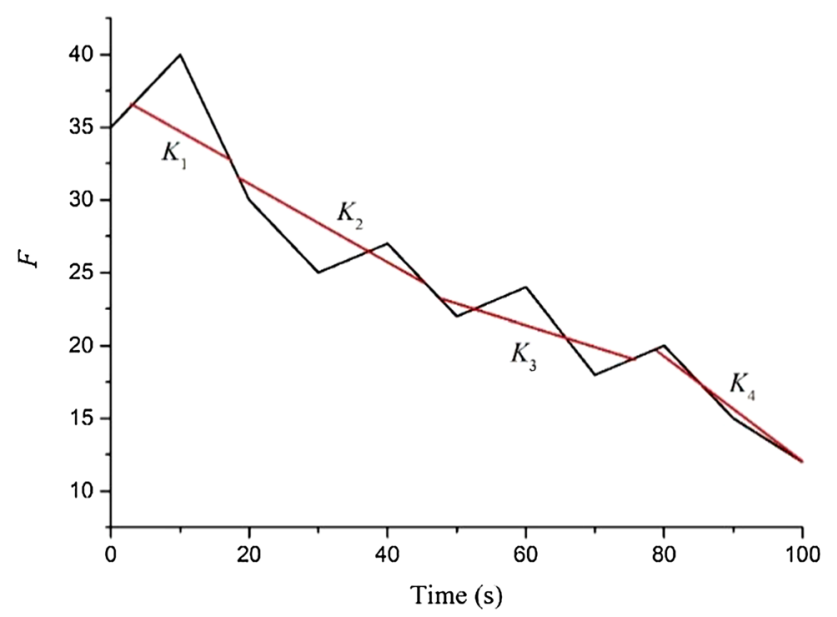

Fig. 4 Solution of slope for energetic exponent curve of the vertical shiplift, the variable range of its parameters implies: $a \in[30.0 \mathrm{~m}, 40.0 \mathrm{~m}], C \in\left[1.0 \times 10^{9} \mathrm{~N} / \mathrm{m}\right.$, $\left.2.0 \times 10^{9} \mathrm{~N} / \mathrm{m}\right], R \in[1.8 \mathrm{~m}, 2.6 \mathrm{~m}]$, respectively. In the corresponding range of values, a series of nodes are selected at the same distance, and the corresponding value $K$ of each node is solved by the improved energetic exponent method.

The influence of distance for lifting point $a$ on energetic exponent $K$ is shown in Fig. 5. The increasing lifting point leads to the increasing reverse overturning moment caused by tension difference of wire ropes at the two lifting points and the increasing pitching acceleration of ship chamber, contributing to the increasing anti overturning capability of ship chamber. The changing range for the absolute value of energetic exponent is 5.00-12.80. Therefore, the energetic exponent decreases with the increasing distance of lifting point a, contributing to the increasing stability.

The influence for stiffness of synchronous shaft $C$ on energetic exponent $K$ is shown in Fig. 6. The synchronous synchronous shaft stiffness increases with increasing reverse torsional moment, contributing to the increasing reverse rotation angular acceleration at the two lifting points, and the rotation speed of the hoist rapidly decays to zero. The changing range for the absolute maximum absolute value of energetic exponent is $4.50 \sim 9.50$. Thus, the energetic exponent decreases with increasing synchronous synchronous shaft stiffness $C$, leading to the increasing stability.

The influence of distance for lifting point $a$ on energetic exponent $K$ is shown in Fig. 7. The lower reverse rotation angular acceleration is attributed to the increasing moment of inertia of hoist caused by the increasing hoist radius when the reverse torsional moment of synchronous shaft is constant. The changing range for the maximum absolute value of energetic exponent is 1.87-3.20. Consequently, the instability enhances with the extending

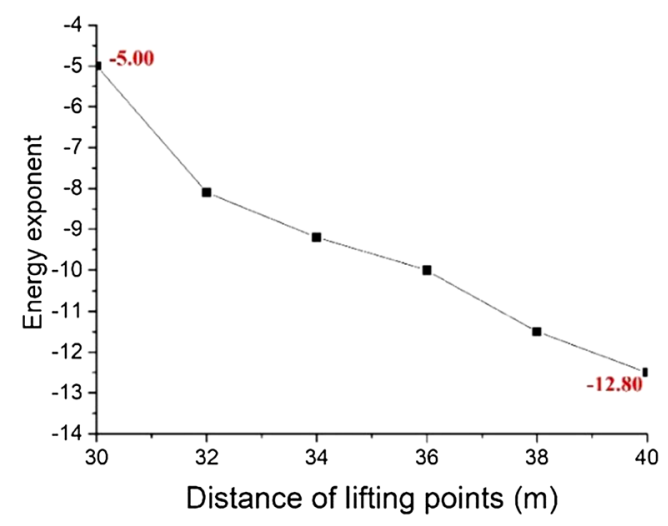

Fig. 5 Influence of distance for lifting point a on energetic exponent $K$ 


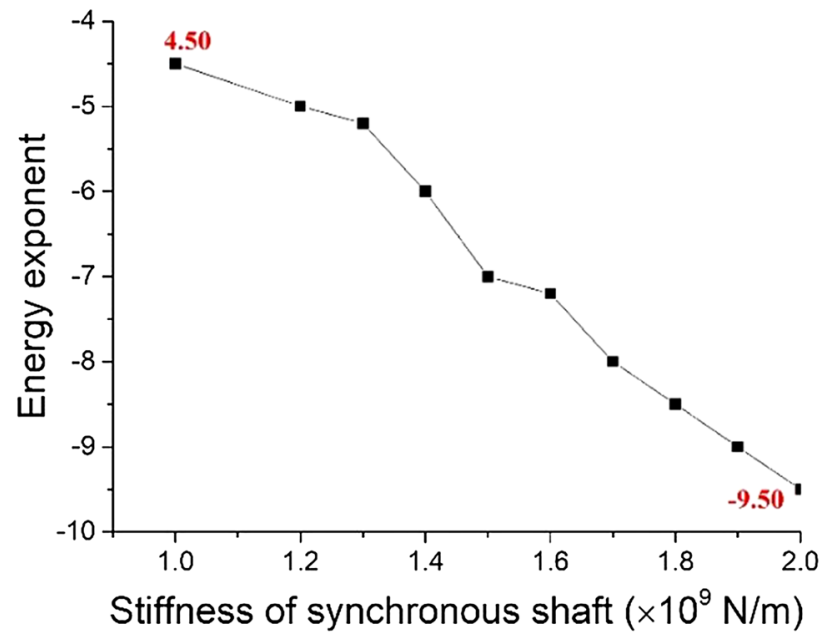

Fig. 6 Influence of synchronous synchronous shaft stiffness $C$ on energetic exponent $K$ decay time of the rotation angular speed for hoist at the two lifting points.

\section{Optimization of shiplift}

With the aim of the avoiding complicated for the optimization of the shiplift, an approximated mathematical model of the energetic exponent slope $K$ is obtained to bring a great convenience for the engineering design. The five data points are selected for each structural parameter according to the equal distance, and a $5 \times 5 \times 5$ three dimensional data set is formed by crossing each other. In this set, based on each group of $a, C, R$, the corresponding $\xi_{1}$ is calculated in accordance of Eq. (16). A function is obtained by using the three dimensional fitting, in the form:

$\xi_{1}=0.07524+2.052 \times 10^{-4} a-3.867 \times 10^{-5} R+2.587 \times 10^{-14} C$.

In the change of time $t$ range $[0,100]$, the energetic exponent slope $K$ is assumed to the following two equations:

$$
\begin{aligned}
& K_{1}=-\frac{1}{3}\left[\frac{F\left(10 n, \xi_{1}\right)-F\left(0, \xi_{1}\right)}{10 n}+\frac{F\left(100-10 n, \xi_{1}\right)-F\left(10 n, \xi_{1}\right)}{100-20 n}+\frac{F\left(100, \xi_{1}\right)-F\left(100-10 n, \xi_{1}\right)}{10 n}\right] \\
& K_{2}=-\frac{1}{4}\left[\frac{F\left(10 n, \xi_{1}\right)-F\left(0, \xi_{1}\right)}{10 n}+\frac{F\left(100-10 n, \xi_{1}\right)-F\left(10 n, \xi_{1}\right)}{50-10 n}+\frac{F\left(100, \xi_{1}\right)-F\left(100-10 n, \xi_{1}\right)}{10 n}\right] .
\end{aligned}
$$

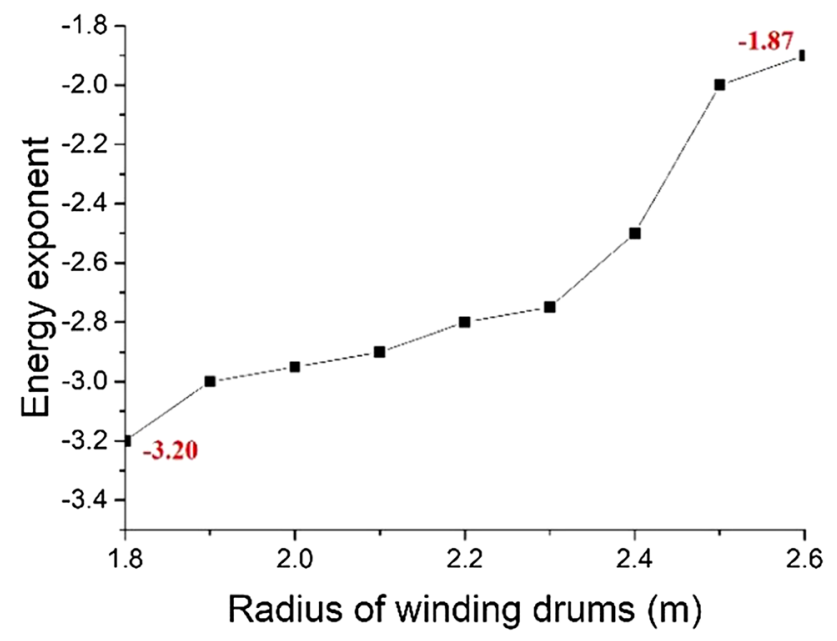

Fig. 7 Influence of radius of winding drums $R$ on energetic exponent $K$

After taking $n=1,2,3,4$, respectively, Table 1 illustrates the difference of the results from Eq. (20) and the improved energetic exponent method, and displays that the error is the lowest when $n=4,3.8 \%$. Therefore, founded on Eqs. (12), (13) and (20b), the approximated mathematical model of the energetic exponent slope is arranged in the form:

$K_{2}=\frac{-1}{160}\left[\ln \left(\frac{I_{1} A_{1}+I_{2} A_{2}}{4 I_{1} h^{6}}\right)+4 \ln \left(\frac{I_{1} A_{3}+I_{2} A_{4}}{I_{1} A_{1}+I_{2} A_{2}}\right)+\ln \left(\frac{I_{1} A_{5}+I_{2} A_{6}}{I_{1} A_{3}+I_{2} A_{4}}\right)\right]$

Table 1 Results of for critical distance of lifting points of seven most representative full balanced hoist vertical shiplift in China

\begin{tabular}{lllll}
\hline Parameters & $n=1$ & $n=2$ & $n=3$ & $n=4$ \\
\hline$K_{1}$ & -3.402 & -1.587 & -0.975 & -0.645 \\
Error & $580.4 \%$ & $217.4 \%$ & $95 \%$ & $29 \%$ \\
$K_{2}$ & -3.320 & -1.501 & -0.882 & -0.519 \\
Error & $564.0 \%$ & $200.2 \%$ & $76.4 \%$ & $3.8 \%$ \\
\hline
\end{tabular}




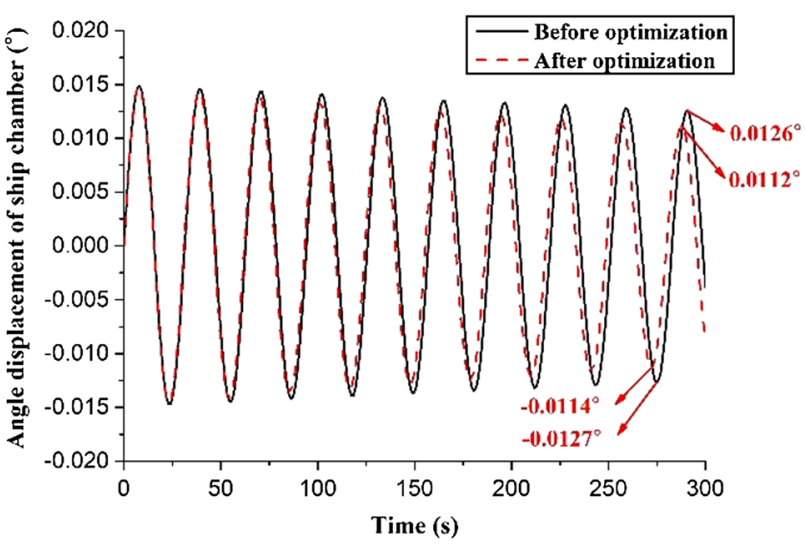

Fig. 8 Responses of pitching angular displacements for ship chamber $a$

$$
\begin{aligned}
& A_{1}=\left[2 e^{40 \xi_{1}}\left(0.643 \xi_{1}+0.766\right) h^{3}\right]^{2} \\
& A_{2}=\left[1.286 e^{40 \xi_{1}} \xi_{1} h^{3}\right]^{2} \\
& A_{3}=\left[2 e^{60 \xi_{1}}\left(0.866 \xi_{1}+0.5\right) h^{3}\right]^{2} \\
& A_{4}=\left[1.732 e^{60 \xi_{1}} \xi_{1} h^{3}\right]^{2} \\
& A_{5}=\left[2 e^{100 \xi_{1}}\left(0.985 \xi_{1}-0.174\right) h^{3}\right]^{2} \\
& A_{6}=\left[1.970 e^{100 \xi_{1}} \xi_{1} h^{3}\right]^{2} .
\end{aligned}
$$

By using Eq. (19), for the enhancement of pitching stability, taking $K_{2}(a, C, R)$ as the objective function, the minimum $K_{2}$ can be obtained. The optimal boundary conditions are written as:

$a \in[30.0,40.0]$

$C \in\left[1.0 \times 10^{9}, 2.0 \times 10^{9}\right]$

$R \in[1.8,2.6]$

Finally, the minimization of the objective function $K_{2}$ $(a, C, R)$ is -15.18 , with $a=40.0 \mathrm{~m}, C=2.0 \times 10^{9} \mathrm{~N} / \mathrm{m}$, and $R=1.8 \mathrm{~m}$.

\section{Numerical validation}

In order to identify the difference of the dynamic characteristics before and after optimization of structural parameters, the further validation is performed for the influence of primary structural parameters on the pitching stability, and the displacement responses are analyzed by the application of the Runge-Kutta algorithm. Figures 8 and 9 illustrate the change of the pitching angular displacement $a$ of ship chamber and the pitching angular displacement $\theta$ of free surface for the second step of the shiplift of GouPitan water conservancy before and after optimization, respectively. The black solid lines in the figures represent the responses of the pitching angular displacement and the pitching angular displacement of free surface before optimization, and the blue solid lines represent that after optimization. As shown in Fig. 8, the last peak of angle displacement close to $300 \mathrm{~s}$ is $0.0126^{\circ}$ before optimization while the value at the same location is $0.0112^{\circ}$ after optimization. The peak of angle displacement is reduced by $11.11 \%$. On the other hand, the last trough of angle displacement close to $300 \mathrm{~s}$ is $-0.0127^{\circ}$ before optimization while the value at the same location is $-0.0114^{\circ}$ after optimization. The absolute values for trough of angle displacement is reduced by $10.24 \%$. For the other three angular displacements, the peak values close to 300 s decrease by $13.33 \%, 15.91 \%, 10.86 \%$ after optimization while absolute values of trough close to $300 \mathrm{~s}$ decrease by $13.16 \%, 15.04 \%, 10.56 \%$ after optimization.

It is revealed that the absolute values of peak and trough of two angular displacements after optimization are smaller than those before optimization. Thus, in the range of time $0 \mathrm{~s}$ to $300 \mathrm{~s}$, the time of dynamic response

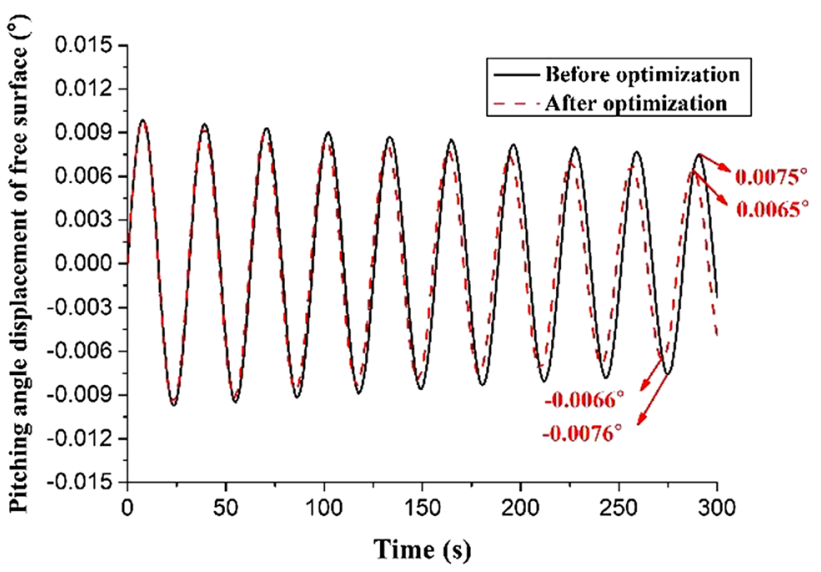

Fig. 9 Responses of pitching angular displacements for free surface $\theta$ 


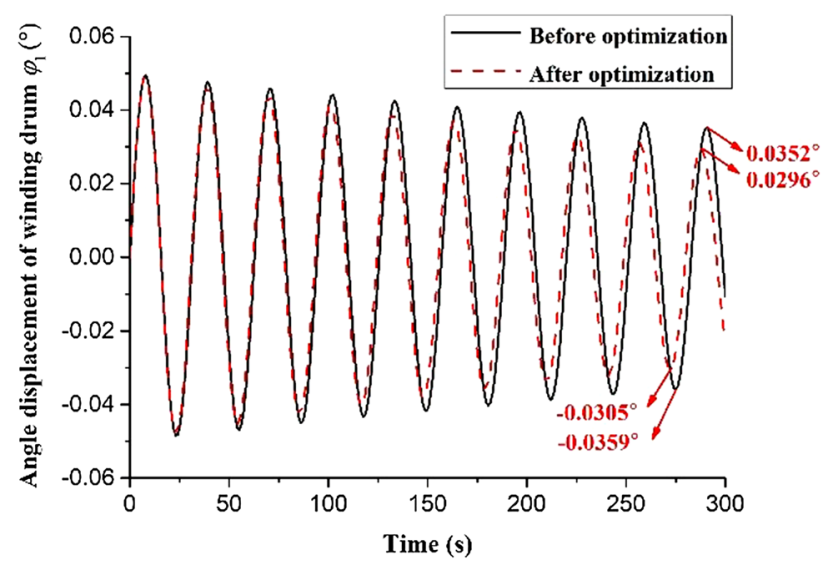

Fig. 10 Responses of angular displacements for winding drum $\varphi_{1}$

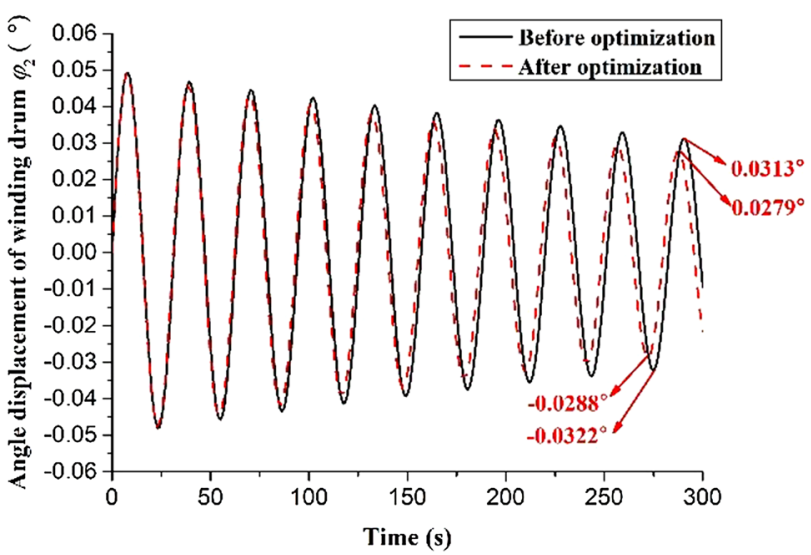

Fig. 11 Responses of angular displacements for winding drum $\varphi_{2}$

Table 2 Influence of primary structural parameters on pitching stability

\begin{tabular}{lll}
\hline \multicolumn{2}{l}{ Parameters } & $t(\mathrm{~s})$ \\
\hline$a(\mathrm{~m})$ & 30.0 & 1.3922 \\
& 35.0 & 0.5849 \\
& 40.0 & 0.5283 \\
$C(\mathrm{~N} / \mathrm{m})$ & $1.0 \times 10^{9}$ & 0.6122 \\
& $1.5 \times 10^{9}$ & 0.6060 \\
& $2.0 \times 10^{9}$ & 0.6035 \\
$R(\mathrm{~m})$ & 1.8 & 0.6062 \\
& 2.3 & 0.6096 \\
& 2.8 & 0.6118 \\
\hline
\end{tabular}

to stability is obviously shortened after optimization, with the evidently-reduced dynamical responses (Figs. 10, 11).

Table 2 presents the influence of the primary structural parameters on the time (the pitching angular displacements decay to $10^{-3}$ degree). The distance of lifting points and the synchronous synchronous shaft stiffness increases gradually with the reducing decaying time. It suggests that the increasing distance of lifting points and synchronous synchronous shaft stiffness arise the more shorten time and increasing stability, Moreover, the hoist radius increases gradually with the increasing decaying time. It indicates that the increasing hoist radius arises the more extended time and decreasing stability.

\section{Conclusion}

In accordance with the 2D fluid solid coupling of ship chamber and mechanical dynamic of shiplift, an improved energetic exponent method founded on the linear water theory, best square approximation method, and the subsection averaging method is presented. In order to pursue the minimum of energetic exponent, the primary structural parameters of shiplift is optimized. Firstly, using the orthogonal polynomial approximation, and the subsection averaging method, an improved energetic exponent method is proposed to analyze the change of stability for shiplift. Based on the improved energetic exponent method, a formulation is presented to calculate the slope of the energetic exponent curve. For the second step of shiplift of GouPitan water conservancy, the error of the formulation in comparison to the improved energetic exponent method, only $3.80 \%$. Moreover, for the enhancement of pitching stability of shiplift, the primary structural parameters of the second step of shiplift of GouPitan water conservancy is optimized to be: $a=40.0 \mathrm{~m}, R=1.8 \mathrm{~m}$, $C=2.0 \times 10^{9} \mathrm{~N} / \mathrm{m}$. Finally, the stability of shiplift increases with an increasing distance of lifting points and synchronous shaft stiffness, while decreases with an increasing hoist radius. Particularly, distance of two lifting points is the most significant factor to affect the stability.

However, the influence of tower column and hydraulic leveling system are not considered in establishing dynamical system. Future work can focus on discussing the stability of shiplift by a mechanical-hydraulic-structural-fluid coupling system.

\section{Compliance with ethical standards}

Conflict of interest The authors declare they have no conflict of interest.

\section{References}

1. Liao L (2014) Safety analysis and design of full balanced hoist vertical shiplifts. Struct Eng Mech 49(3):311-327

2. Cheng X, Shi D, Li H, Xia R, Zhang Y, Zhou J (2018) Stability and parameters influence study of Fully Balanced Hoist Vertical Ship Lift. Struct Eng Mech 66(5):583-594 
3. Michael B (2014) 75 Jahre Schiffshebewerk in Rothensee. Bautechnik 91:380-387

4. Chen J, Ma G (1996) Chamber stability of hoisting fully balancing type vertical ship lift. Hydro-Sci Eng 4:301-308

5. Cheng G, Li H, Ruan S (2005) Free vibration characteristics and stability analysis of ship lift system. J Mech Strength 3:276-281

6. Ruan S, Cheng G (2003) Calculation of ship-liquid-chamber coupled system in the ship lift with finite element method in time domain. Chin J Comput Mech 3:290-294

7. Shi D, Shong Z, Liao L (2003) Coupling analysis of ship-chamber of ship lift and parametric vibration of liquid. Eng J Wuhan Univ 1:77-80

8. Pearlson DL (1985) Installation of syncrolift equipment in ports. In: Port engineering and operation, proceedings of the conference on British ports and their future, pp 177-189

9. Nakayama T, Washizu K (1980) Nonlinear analysis of liquid motion in a container subjected to forced pitching oscillation. Int J Numer Meth Eng 8:1207-1220

10. Zhang Y, Shi D, Shi L, Xia R, Cheng X, Zhou J (2019) Analytical solution of capsizing moments in ship chamber under pitching excitation. Proc Inst Mech Eng Part C J Mech Eng Sci 233(15):5294-5301

11. Zhang Y, Shi D, Shi L, Xia R, Cheng X (2019) Pitch stability analysis of high-lift wire ropes hoist vertical shiplift under shallow water sloshing-structure interaction. Proc Inst Mech Eng Part K J Mutibody Dyn 233(4):942-955

12. Benjamin T, Ursell F (1954) The stability of the plane free surface of a liquid in vertical periodic motion. Proc R Soc London Ser A Math Phys Sci 225:505-515

13. Peric M, Zorn T, Moctar O, Schellin T, Kim Y (2009) Simulation of sloshing in LNG-tanks. J Offshore Mech Arct Eng Trans ASME 131(3):031101-11
14. Ushijima S (1998) Three-dimensional arbitrary Lagrangian-Eulerian numerical prediction method for non-linear free surface oscillation. Int J Numer Methods Fluids 26:605-623

15. Wang C, Khoo B (2005) Finite element analysis of two dimensional nonlinear sloshing problems in random excitations. Ocean Eng 32(2):107-133

16. Horsley D, Forbes $L$ (2013) A spectral method for Faraday waves in rectangular tanks. J Eng Math 79(1):13-33

17. Faltinsen $O$ (1974) A nonlinear theory of sloshing in rectangular tanks. J Ship Res 18(4):114-241

18. Faltinsen $O$ (1978) A numerical nonlinear method of sloshing in tanks with two-dimensional flow. J Ship Res 22(3):193-202

19. Faltinsen O, Rognebakke O, Lukovsky I, Timokha A (2000) Multidimensional model analysis of nonlinear sloshing in a rectangular tank with finite water depth. J Fluid Mech 407:201-234

20. Ibrahim R, Barr A (1978) Parametric vibration, part IV: current problem (2). Shock Vib 10(4):19-47

21. Faltinsen O, Rognebakke O, Timokha A (2003) Resonant threedimensional nonlinear sloshing in a square-base basin analysis of nonlinear sloshing in a rectangular tank. J Fluid Mech 487:1-42

22. Allgower E, Georg K (2003) Numerical continuation methods: an introduction. Springer Ser Comput Math 13(199):388

23. Li Y, Wang Z (2015) Unstable characteristics of two-dimensional parametric sloshing in various shape tanks: theoretical and experimental analysis. J Vib Control 22(19):4025-4046

24. Li Y, Wang L, Yu YQ (2016) Stability analysis of parametrically excited systems using the energetic-growth exponent/coefficient. Int J Struct Stab Dyn 17(2):1750018

Publisher's Note Springer Nature remains neutral with regard to jurisdictional claims in published maps and institutional affiliations. 